\title{
Genetic Characterization of Japanese Encephalitis Virus Genotype 5 Isolated from Patient, South Korea, 2015
}

Jae Hoon Woo, ${ }^{1}$ Young Eui Jeong, ${ }^{1}$ Jung Eun Jo, Sang-Mu Shim, Jungsang Ryou, Kyung-Chang Kim, Won Ja Lee, Joo-Yeon Lee

We isolated Japanese encephalitis virus genotype 5 from human specimens in South Korea. Whole-genome analysis showed $90.4 \%$ identity with other genotype 5 viruses from humans. This virus had a unique insertion in the NS4A gene. However, the envelope protein contained Lys 84, which was specific to strains of genotype 5 viruses from South Korea.

Tapanese encephalitis is caused by Japanese encephalitis virus (JEV), a mosquitoborne virus of the family Flaviviridae, genus Flavivirus (1). The JEV genome is composed of a single-stranded, positive-sense RNA of $\approx 11 \mathrm{~kb}$ with a single open reading frame (ORF) encoding a polyprotein. The polyprotein is processed into 3 structural proteins, capsid, membrane, and envelope (E), and 7 nonstructural proteins, NS1, NS2A, NS2B, NS3, NS4A, NS4B, and NS5 (2).

$\mathrm{JEV}$ is distributed in temperate and tropical areas of eastern and Southeast Asia. In 2010, JEV genotype 1 was the predominant virus circulating. However, genotype 5 was also identified in mosquitoes in South Korea (3). Since that time, JEV genotype 5 has been detected in mosquitoes in many areas of South Korea (4). We report isolation of JEV genotype 5 virus from patient specimens and differences in sequences among other JEV strains (genotypes 1-5).

\section{The Study}

We isolated JEV (strain K15P38) from samples of a 27-year-old woman who came to a hospital in Kyeonggi-do, South Korea, on November 8, 2015.

Author affiliations: Centers for Disease Control and Prevention, Chongju, South Korea (J.H. Woo, Y.E. Jeong, J.E. Jo, S.-M. Shim, J. Ryou, K.-C. Kim, W.J. Lee, J.-Y. Lee); MIRIMEDIX Corporation, Gangwon, South Korea (Y.E. Jeong)

DOI: https://doi.org/10.3201/eid2605.190977
The patient had mild symptoms, such as fever, headache, apathy, and nausea. The patient recovered. We obtained documentation from the hospital that she had been vaccinated against Japanese encephalitis. Cerebrospinal fluid (CSF) and serum samples were obtained during the acute and convalescent phases.

We detected JEV IgM in serum and CSF samples by using an ELISA (Inbios, https://inbios.com) for convalescent-phase samples, but not acute-phase samples. We isolated virus by inoculating the convalescent-phase CSF sample onto BHK-21 cells. After a cytopathic effect was observed, we confirmed presence of virus by using a quantitative real-time PCR. We performed whole-genome sequence analysis of the virus by using virus genome extracted from 5 passaged culture supernatants and QIAamp Viral RNA Mini Kit (QIAGEN, https:/ / www.qiagen.com).

We performed next-generation sequencing for full-length genes by using the Illumina (https:// www. illumina.com) and confirmed gaps from next-generation sequencing by using Sanger sequencing. We assembled nucleotide sequences by using the SeqMan program in DNASTAR software version 5.06 (https://www. dnastar.com). We then conducted molecular phylogenetic analysis of ORF nucleotide sequences with 30 previously reported JEV strains by using MEGA 6.0 software (https://www.megasoftware.net) and the maximum-likelihood method (5) and calculated timescale phylogenies by using BEAST version 2.6.0 software (6). We deposited the polyprotein genome sequence of strain K15P38 in GenBank (accession no. MK541529).

We compared the entire ORF sequences of K15P38 virus with previously reported strains of JEV genotypes 1-5. Phylogenetic analysis showed that K15P38 belonged to JEV genotype 5 by (Figures 1, 2,

${ }^{1}$ These authors contributed equally to this article. 


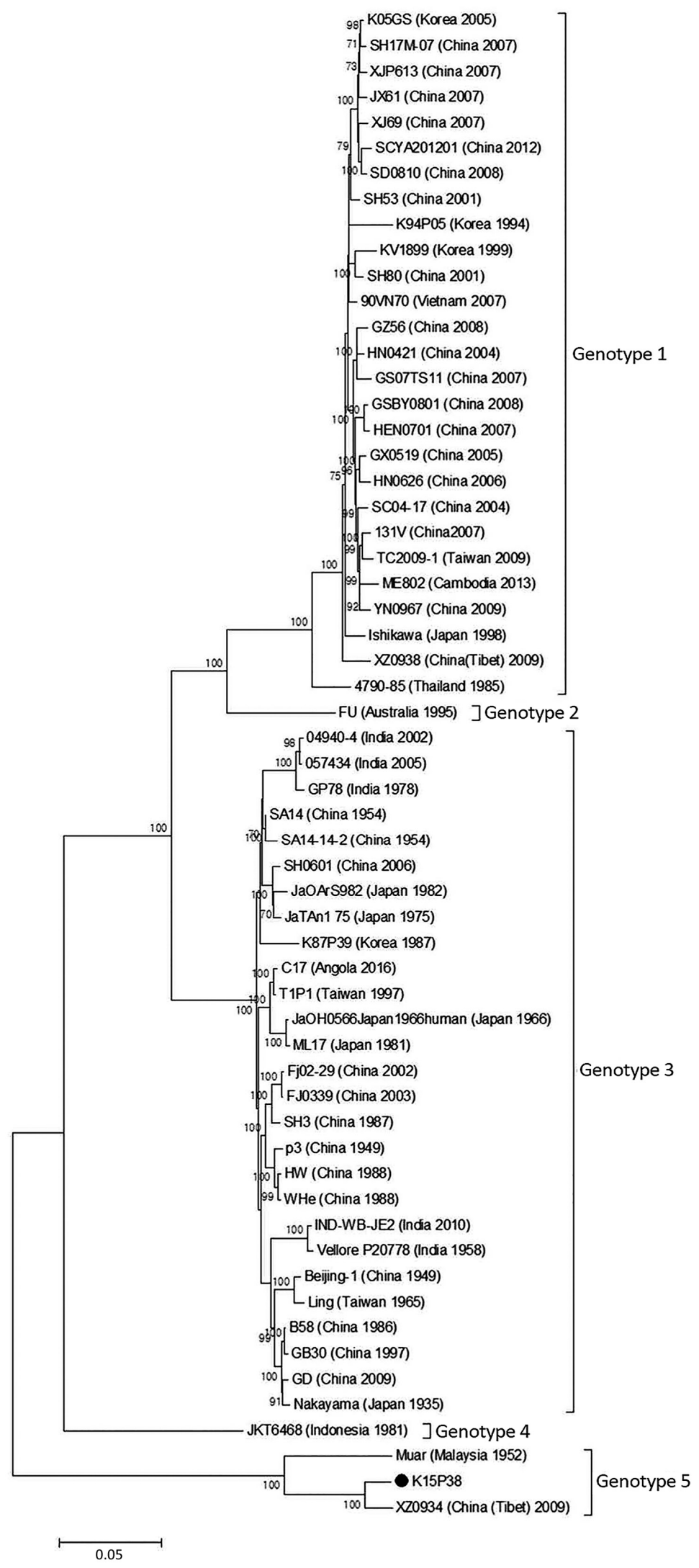

Figure 1. Phylogenetic tree of Japanese encephalitis virus genotypes 1-5, South Korea. Entire open reading frame is shown. Bootstrap probabilities (values along branches) of each node were calculated by using 1,000 replicates. Branches showing quartet puzzling reliability $>70 \%$ can be considered well supported. Black circle indicates K15P38 strain from patient samples. Scale bar indicates nucleotide substitutions per site. 


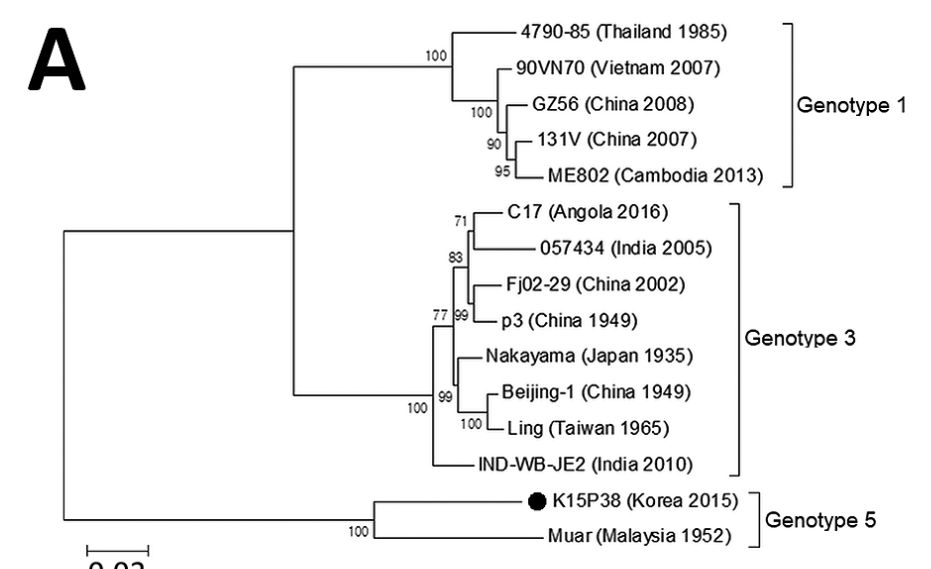

$\overleftrightarrow{0.02}$

B

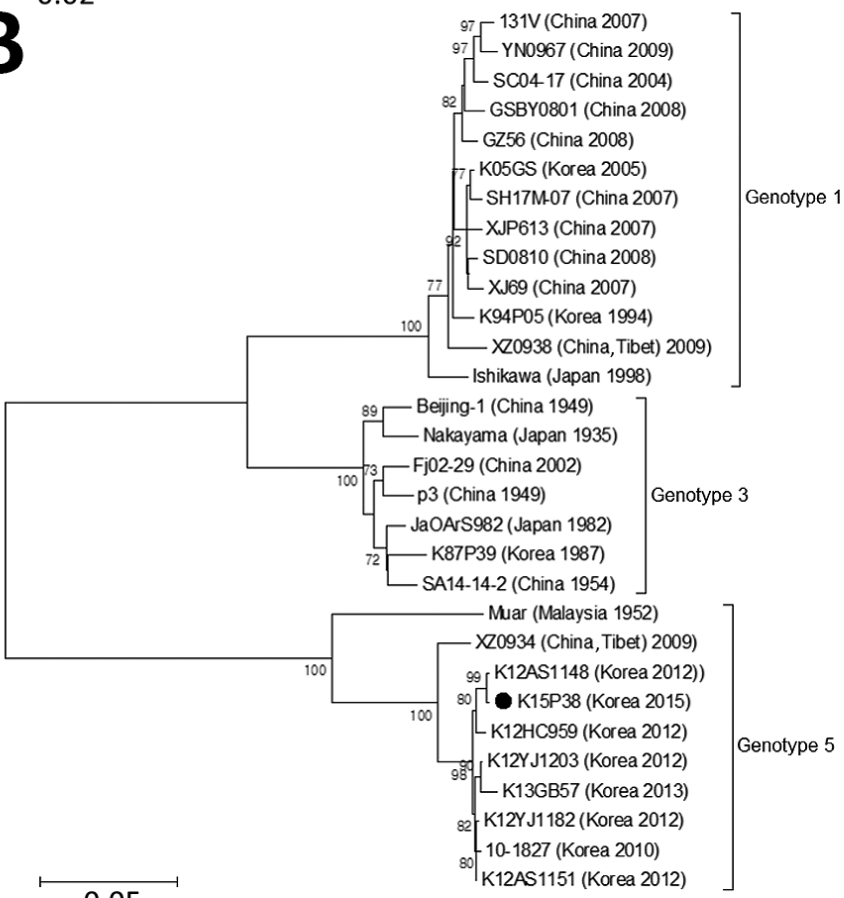

C

0.05

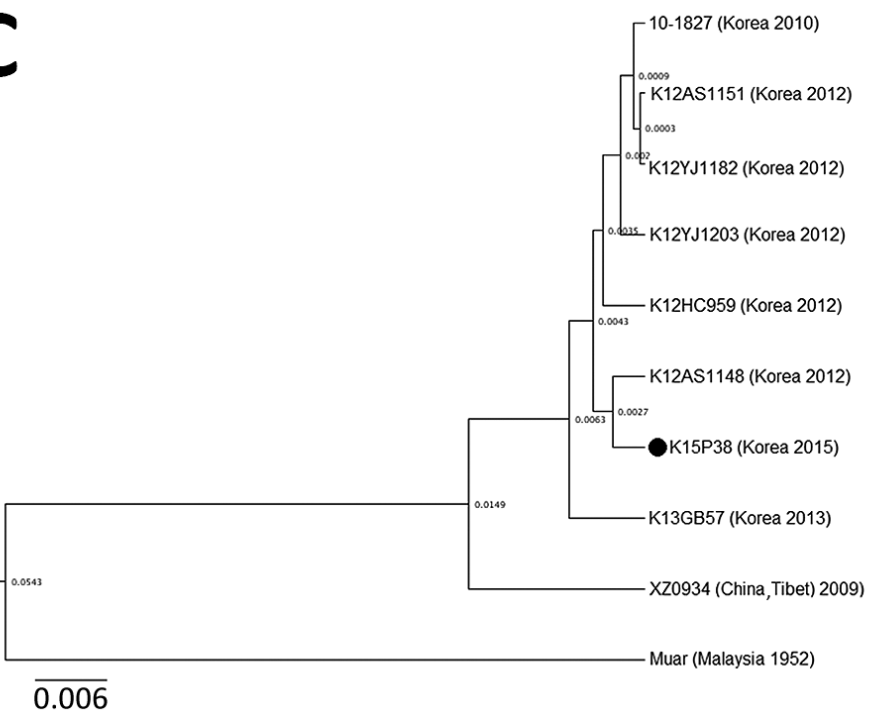

Figure 2. Phylogenetic trees of Japanese encephalitis virus (JEV) genotypes 1,3 , and 5 , South Korea. A) Entire open reading frame of JEV human isolates. B) Envelope protein genes of JEV human isolates. C) Divergence time estimation based on the envelope protein genes of JEV genotype 5. Bootstrap probabilities (values along branches) of each node were calculated by using 1,000 replicates. Branches showing quartet puzzling reliability $>70 \%$ can be considered well supported. Black circles indicate K15P38 strain from patient samples. Scale bars indicate nucleotide substitutions per site. 
Japanese Encephalitis Virus Genotype 5, South Korea

Table 2. Comparison of amino acid sequences of envelope protein of Japanese encephalitis viruses of genotype 5 , South Korea*

\begin{tabular}{|c|c|c|c|c|c|c|c|c|c|c|c|c|c|}
\hline \multirow[b]{2}{*}{ Virus } & \multicolumn{13}{|c|}{ Amino acid position } \\
\hline & 42 & 52 & 58 & 84 & 129 & 156 & 161 & 171 & 208 & 240 & 292 & 343 & 473 \\
\hline K15P38 (South Korea 2015) & $\mathrm{D}$ & $\mathrm{E}$ & $\mathrm{T}$ & $\mathrm{K}$ & I & $T$ & A & $\mathrm{P}$ & $S$ & $\mathrm{M}$ & $\mathrm{E}$ & A & I \\
\hline 10-1827 (South Korea 2010) & . & . & . & . & . & . & & . & . & . & . & . & . \\
\hline K12AS1148 (South Korea 2012) & . & . & . & . & . & . & . & . & . & . & . & . & . \\
\hline K12AS1151 (South Korea 2012) & . & . & & . & & . & . & . & . & . & . & $\therefore$ & . \\
\hline K12HC95 (South Korea 2012) & . & . & A & . & $\mathrm{T}$ & . & . & . & . & . & . & V & . \\
\hline K12YJ1182 (South Korea 2012) & . & . & . & . & . & . & . & . & . & . & . & . & . \\
\hline K12YJ1203 (South Korea 2012) & & . & . & . & & . & & L & . & . & . & . & \\
\hline K13GB57 (South Korea 2013) & G & . & . & 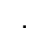 & . & & V & . & . & . & . & . & $\mathrm{T}$ \\
\hline Muar (Malaysia 1952) & . & Q & . & $\mathrm{R}$ & . & S & . & . & $\mathrm{T}$ & L & D & . & . \\
\hline XZ0934 (China (Tibet) 2009) & . & . & . & $\mathrm{R}$ & . & . & . & . & . & . & . & . & . \\
\hline
\end{tabular}

panel A; Appendix Table 1, https://wwwnc.cdc. gov/EID/article/26/5/19-0977-App1.pdf). Identities between the entire ORF of K15P38 and Muar genotype 5 virus were $90.4 \%$.

In general, the E gene of JEV plays a major role in the pathogenesis of encephalitis (7). Several amino acids, including 107, 138, and 176 in the E protein, are reported to play major roles in the neurovirulence of JEV. K15P38 virus had conserved amino acids at these sites $(8,9)$. However, the E protein of this virus had 6 different amino acids compared with that of the Muar strain isolated from a human in Malaysia in 1952 (10). Because Muar virus was derived from mouse brain and $\mathrm{K} 15 \mathrm{P} 38$ virus was passaged in cell culture, we do not exclude the possibility of sequence variation caused by different culture methods.

Furthermore, the K15P38 strain contained Lys rather than Arg at position 84 of the E protein (Table 2 ), which was unique in genotype 5 viruses from South Korea strains derived from mosquito and human specimens. The E gene sequences of K15P38 virus showed high identity of $\approx 98.5 \% 99.8 \%$ with other genotype 5 strains from South Korea isolated from mosquitoes (Figure 2, panel B). By estimating the root of the time measured on the $\mathrm{E}$ gene of JEV genotype 5 viruses, we identified that the XZ0934 strain from Tibet was an ancestor of JEV genotype 5 virus strains from South Korea (Figure 2, panel C). Considering these variations and time estimation of JEV genotype 5, further study is needed to investigate molecular and biologic characteristics of JEV.

\section{Conclusions}

JEV genotype 5 was isolated from mosquitoes in China during 2009 and South Korea during 2010. Because the major JEV genotype from mosquitoes in South Korea changed from genotype 1 to genotype 5 during 2010, the number of infected patients increased coincidently, especially adult patients $(8,11)$. Japanese encephalitis is generally more prevalent in southern areas of South Korea, wherein Culex tritaeniorhynchus mosquitoes are more prevalent than in other regions. However, the prevalence of Japanese encephalitis has also increased in northern regions of South Korea, including Seoul, Gyeonggi, and Gangwon since 2010. This finding is consistent with the fact that $5 \mathrm{JEVs}$ with genotype 5 have been reported in more diverse mosquito species, including $C x$. orientalis and $C x$ pipiens, not only in Cx. tritaeniorincus (4).

A previous study showed that the prevalence of neutralizing antibodies to JEV were maintained at a level of $98.1 \%$ among the general population (12) because of the National Vaccine Program against Japanese encephalitis in South Korea since 1982. The currently used Japanese encephalitis vaccine that contains the JEV genotype 3 strain provides adequate protection against JEV genotype 1 (13).

Even so, the number of adult patients with Japanese encephalitis has increased. It has also been reported that existing JEV genotype 3 vaccines are less effective in protecting against JEV genotype 5 (14), suggesting the need for studies of the protective effect of current Japanese encephalitis vaccine against JEV genotype 5 virus.

Although JEV genotype 5 is highly pathogenic and causes early viremia and central nervous system invasion in animal models, limited information is available on the biological nature of JEV G5. Our results provide potentially useful information regarding JEV genotype 5, including pathogenic characteristics and vaccine efficacy.

This study was supported by the Korea National Institute of Health, Korea Centers for Disease Control and Prevention (grants nos. 4845-300-210-13 and 4837-301-210-13).

\section{About the Author}

Mr. Woo is a senior research scientist in the Division of Emerging Infectious Disease and Vector Research, National Institute of Health, Korea Centers for Disease Control and Prevention, Chongju, South Korea. His 
primary research interest is flaviviruses, including Japanese encephalitis virus, West Nile virus, and Zika virus.

\section{References}

1. Vaughn DW, Hoke CH Jr. The epidemiology of Japanese encephalitis: prospects for prevention. Epidemiol Rev. 1992;14:197-221. https://doi.org/10.1093/oxfordjournals. epirev.a036087

2. Chambers TJ, Hahn CS, Galler R, Rice CM. Flavivirus genome organization, expression, and replication. Annu Rev Microbiol. 1990;44:649-88. https://doi.org/10.1146/annurev. mi.44.100190.003245

3. Takhampunya R, Kim HC, Tippayachai B, Kengluecha A, Klein TA, Lee WJ, et al. Emergence of Japanese encephalitis virus genotype V in the Republic of Korea. Virol J. 2011;8:449. https://doi.org/10.1186/1743-422X-8-449

4. Kim H, Cha GW, Jeong YE, Lee WG, Chang KS, Roh JY, et al. Detection of Japanese encephalitis virus genotype $\mathrm{V}$ in Culex orientalis and Culex pipiens (Diptera: Culicidae) in Korea. PLoS One. 2015;10:e0116547. https:/ / doi.org/10.1371/ journal.pone.0116547

5. Schmidt HA, Strimmer K, Vingron M, von Haeseler A. TREE-PUZZLE: maximum likelihood phylogenetic analysis using quartets and parallel computing. Bioinformatics. 2002;18:502-4. https:/ / doi.org/10.1093/ bioinformatics/18.3.502

6. Drummond AJ, Rambaut A. BEAST: Bayesian evolutionary analysis by sampling trees. BMC Evol Biol. 2007;7:214. https://doi.org/10.1186/1471-2148-7-214

7. Yu Y. Phenotypic and genotypic characteristics of Japanese encephalitis attenuated live vaccine virus SA14-14-2 and their stabilities. Vaccine. 2010;28:3635-41. https://doi.org/ 10.1016/j.vaccine.2010.02.105

8. Arroyo J, Guirakhoo F, Fenner S, Zhang ZX, Monath TP, Chambers TJ. Molecular basis for attenuation of neurovirulence of a yellow fever Virus/Japanese encephalitis virus chimera vaccine (ChimeriVax-JE). J Virol. 2001;75:93442. https:// doi.org/10.1128/JVI.75.2.934-942.2001

9. Yang J, Yang H, Li Z, Wang W, Lin H, Liu L, et al. Envelope protein mutations L107F and E138K are important for neurovirulence attenuation for Japanese encephalitis virus SA14-14-2 strain. Viruses. 2017;9:E20. https:// doi.org/ 10.3390/v9010020

10. Solomon T, Ni H, Beasley DW, Ekkelenkamp M, Cardosa MJ, Barrett AD. Origin and evolution of Japanese encephalitis virus in southeast Asia. J Virol. 2003;77:3091-8. https://doi.org/10.1128/JVI.77.5.3091-3098.2003

11. Yun SM, Cho JE, Ju YR, Kim SY, Ryou J, Han MG, et al. Molecular epidemiology of Japanese encephalitis virus circulating in South Korea, 1983-2005. Virol J. 2010;7:127. https://doi.org/10.1186/1743-422X-7-127

12. Lee EJ, Cha GW, Ju YR, Han MG, Lee WJ, Jeong YE. Prevalence of neutralizing antibodies to Japanese encephalitis virus among high-risk age groups in South Korea, 2010. PLoS One. 2016;11:e0147841. https:/ / doi.org/ 10.1371/journal.pone.0147841

13. Van Gessel Y, Klade CS, Putnak R, Formica A, Krasaesub S, Spruth $\mathrm{M}$, et al. Correlation of protection against Japanese encephalitis virus and JE vaccine (IXIARO(®)) induced neutralizing antibody titers. Vaccine. 2011;29:5925-31. https:// doi.org/10.1016/j.vaccine.2011.06.062

14. Cao L, Fu S, Gao X, Li M, Cui S, Li X, et al. Low protective efficacy of the current Japanese encephalitis vaccine against the emerging genotype 5 Japanese encephalitis Virus. PLoS Negl Trop Dis. 2016;10:e0004686. https:/ / doi.org/10.1371/ journal.pntd.0004686

Address for correspondence: Joo-Yeon Lee, Division of Emerging Infectious Disease and Vector Research, Center for Infectious Disease Research National Research Institute of Health, Centers for Disease Control and Prevention, 187 Osongsaengmyeong2-ro, Osong-eup, Heungdeok-gu, Cheongju-si, Chungcheongbuk-do, 28159, South Korea; email: ljyljy@nih.go.kr 